\title{
ON CERTAIN SUBCLASSES OF MEROMORPHICALLY MULTIVALENT FUNCTIONS INVOLVING A LINEAR OPERATOR
}

\author{
J. PATEl And A. Ku. PAlit
}

Abstract. The purpose of the present paper is to derive some inclusion relationships and other interesting properties of a certain subclass $\Sigma_{p}^{+}(a, c, A, B)$ of meromorphically p-valent functions with positive coefficients which are defined by means of a linear operator. The familiar concept of neighborhood of analytic functions is extended and applied to meromorphically p-valent functions considered here. We also derive many interesting results on the Hadamard product of functions belonging to the class $\Sigma_{p}^{+}(a, c, A, B)$.

Mathematics subject classification (2010): 30C45.

Keywords and phrases: Meromorphic functions, differential subordination, Hadamard product (or convolution), neighborhoods, linear operator.

\section{REFERENCES}

[1] O. Altintas, Neighborhoods of certain analytic functions with negative coefficients, Int. J. Math. Math. Sci., 19 (1996), 797-800.

[2] O. Altintas, Ö. ÖZAKAN, H.M. SRIVASTAVA, Neighborhoods of a class of analytic functions with negative coefficients, Appl. Math. Lett., 13 (2000), 63-67.

[3] M. K. AouF, On a class of meromorphic multivalent functions with positive coefficients, Math. Japonica, 35 (1990), 603-608.

[4] M.K. AouF, A generalization of meromorphic multivalent functions with positive coefficients, Math. Japonica, 35 (1990), 609-614.

[5] M. K. Aouf, Certain subclasses of meromorphically multivalent functions associated with generalized hypergeometric function, Comput. Math. Appl., 55 (2008), 494-509.

[6] N. E. CHO, M. NunOKAWA, On certain subclasses meromorphically multivalent functions, Chinese J. Math., 22 (1994), 197-202.

[7] M. D. Ganigi, B.A. Uralegaddi, Subclasses of meromorphic close-to-convex functions, Bull. Math. Soc. Sci. Math. Roumanie (N.S.), 33, 81 (1989), 105-109.

[8] A. W. Goodman, Univalent functions and nonanalytic functions, Proc. Amer. Math. Soc., 8 (1975), 598-601.

[9] J.-L. LIU, H. M. SRIVASTAVA, A linear operator and associated families of meromorphically multivalent functions, J. Math. Anal. Appl., 259 (2001), 566-581.

[10] M. L. MogRA, Meromorphic multivalent functions with positive coefficients, I \& II, Math. Japonica, 35 (1990), 1-11 and 1089-1098.

[11] B. A. URAlegaddi, M. D. GANigi, Meromorphic multivalent functions with positive coefficients, Nepali Math. Sci. Rep., 11, 2 (1986), 95-102.

[12] B. A. Uralegaddi, C. Somanatha, Certain classes of meromorphic multivalent functions, Tamkang J. Math., 23 (1992), 223-231.

[13] S. Ruscheweyh, Neighborhoods of univalent functions, Proc. Amer. Math. Soc., 81 (1981), $521-$ 527. 